\title{
Las estatinas reducen la mortalidad y los eventos cardiovasculares en pacientes portadores de insuficiencia renal crónica en etapa pre-dialítica
}

Statins reduce cardiovascular events and mortality in pre dialysis chronic kidney disease patients

Palmer S. y col. Annals of Internal Medicine. 2012;157(4):263-275.

\section{Objetivos}

Determinar los beneficios y perjuicios de la terapia a base de estatinas en pacientes portadores de insuficiencia renal crónica (IRC), y examinar si el efecto de estos fármacos varía en función del grado de nefropatía.

\section{Fuente de datos}

Cochrane y EMBASE, hasta febrero 2012 (sin restricción de idioma). Revisión sistemática y meta-análisis.

\section{Selección de estudios}

Ensayos clínicos controlados aleatorizados* en los que se compararon estatinas contra placebo, no tratamiento, cuidado estándar u otra estatina, respecto de mortalidad y eventos car- diovasculares, en pacientes portadores de diversos estadios de IRC (según definición de National Kidney Foundation), publicados hasta julio 2006.

\section{Extracción de datos}

Los ensayos clínicos fueron extraídos de las bases de datos por dos revisores independientes que identificaron el material elegible en función de los criterios de inclusión determinados. Se excluyeron aquellos estudios con menos de ocho semanas de seguimiento.

\section{Resultados Principales}

Los resultados principales se resumen en la tabla 1.

Tabla 1: Reducción de la mortalidad y eventos cardiovasculares en pacientes tratados con estatinas de acuerdo al deterioro de la función renal.

\begin{tabular}{l|c|c|c} 
& Pre-diálisis RR (IC95\%) & Diálisis RR (IC95\%) & Transplante renal RR (IC95\%) \\
\hline Mortalidad General & $0,81(0,74$ a 0,88) & $0,96(0,88$ a 1,04) & $0,68(0,45$ a 1,02) \\
\hline Mortalidad Cardiovascular & $0,78(0,68$ a 0,89) & $0,94(0,82$ a 1,07) & $0,68(0,45$ a 1,02) \\
\hline Eventos Cardiovasculares & $0,76(0,73$ a 0,80) & $0,95(0,87$ a 1,03) & $0,84(0,66$ a 1,06) \\
\hline
\end{tabular}

RR: riesgo relativo* IC: intervalo de confianza*

\section{Conclusión}

Las estatinas reducen la mortalidad y los eventos cardiovasculares en pacientes portadores de IRC en etapa pre-dialítica y poseen bajo o ningún efecto en aquellos que dializan y efecto incierto en aquellos con trasplante renal.

Fuentes de financiamiento: sin financiación externa

\section{Comentario}

La dislipidemia es un desorden muy frecuente en pacientes portadores de IRC, en quienes constituye no sólo un factor de riesgo de enfermedad cardiovascular, sino también de progresión de su nefropatía, dado que el depósito de lípidos a nivel mesangial desencadenaría un proceso inflamatorio local con el consiguiente daño parenquimatoso y deterioro de la función renal. Por dicho motivo, el tratamiento de la dislipidemia forma parte del conjunto de medidas nefroprotectoras. Dichas medidas son extensibles no sólo a los pacientes portadores de IRC pre-dialítica, sino también a los pacientes en diálisis crónica con función renal residual conservada, pues la preservación de dicha función residual se asocia a menor mortalidad, y también a los pacientes trasplantados renales, a fin de contribuir a la sobrevida de su injerto.

En el artículo que hemos resumido, sus autores concluyen que las estatinas reducen la mortalidad y los eventos cardiovasculares en pacientes portadores de IRC en etapa pre-dialítica, pero poseen bajo o ningún efecto en aquellos que dializan y su efecto es incierto en los trasplantados renales. Este fenómeno podría explicarse a raíz de la mayor complejidad que la etiopatogenia de la enfermedad cardiovascular adquiere en etapas avanzadas de la IRC, donde incluso el mismo tratamiento de sustitución renal podría estar jugando un rol en la misma. Así por ejemplo, en el caso de pacientes trasplantados renales, algunas de las drogas inmunosupresoras que reciben para evitar el rechazo del injerto, tales como metilprednisona, sirolimus y tacrolimus, son al mismo tiempo inductoras de dislipidemia. Por el contrario, en pacientes con nefropatía crónica avanzada, los valores séricos bajos de colesterol total y LDL correlacionan con mayor mortalidad. Probablemente, en estos casos, la hipocolesterolemia refleje un estado de desnutrición severa, y la mayor mortalidad documentada sea una consecuencia de la misma.

\section{Conclusión del comentador}

Si bien a la luz de lo concluido por este meta-análisis, la evidencia avalaría el uso de las estatinas fundamentalmente en el tratamiento de la dislipidemia del paciente portador de IRC predialítica, el hecho de que se haya documentado cierto grado de beneficio en pacientes portadores de IRC bajo tratamiento sustitutivo renal (diálisis o trasplante), así como su importancia en las estrategias de nefroprotección, tornan recomendable el uso de estos fármacos para el tratamiento de la dislipidemia en toda la población portadora de nefropatía crónica.

Carlos G. Musso [ Servicio de Nefrología del Hospital Italiano de Buenos Aires. carlos.musso@ hiba.org.ar ]

Musso CG. Las estatinas reducen la mortalidad y los eventos cardiovasculares en pacientes portadores de insuficiencia renal crónica en etapa predialítica. Evid Act Pract Ambul. 2013;16(1): 7. Comentado de: Navaneethan S. y col. Benefits and harms of statin therapy for persons with chronic kidney disease. Annals of Internal Medicine. 2012; 157(4): 263-275. PMID: 22910937.

\section{Referencias}

1. Clarkson M, Magee C, Brenner B. The kidney. Philadelphia. Saunders. 2008: 567-584.

2. Upadhyay A, Earley A, Lamont J, Haynes S, Wanner C, Balk E. Lipid-lowering therapy in persons with chronic kidney disease. Annals of Intemal Medicine. 2012; 157:251-262. 